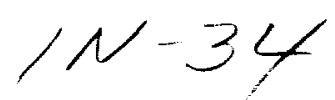

NASA Contractor Report 195047

ICASE Report No. 95-13

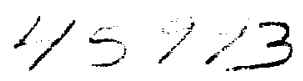

120
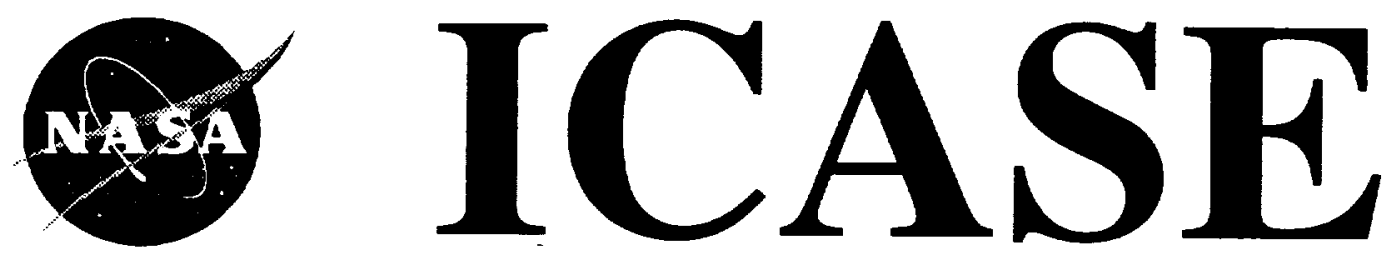

\title{
SPECTRUM AND ENERGY TRANSFER IN STEADY BURGERS TURBULENCE
}

\section{Sharath S. Girimaji}

Ye Zhou

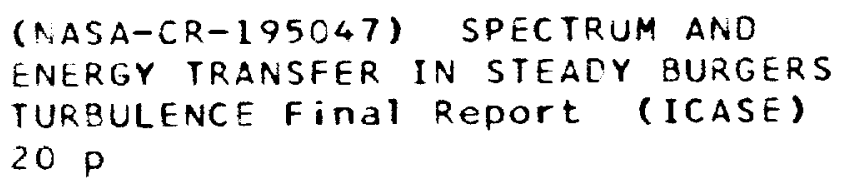

Contract No. NAS1-19480 March 1995

Institute for Computer Applications in Science and Engineering NASA Langley Research Center Hampton, VA 23681-0001

SRA Operated by Universities Space Research Association 


\title{
SPECTRUM AND ENERGY TRANSFER IN STEADY BURGERS TURBULENCE*
}

\author{
Sharath S. Girimaji and Ye Zhou \\ ICASE, NASA Langley Research Center \\ Hampton, VA 23681
}

\begin{abstract}
The spectrum, energy transfer and spectral interactions in steady Burgers turbulence are studied using numerically generated data. The velocity field is initially random and the turbulence is maintained steady by forcing the amplitude of a band of low wavenumbers to be invariant in time, while permitting the phase to change as dictated by the equation. The spectrum, as expected, is very different from that of Navier-Stokes turbulence. It is demonstrated, for the first time, that the far range of the spectrum scales as predicted by Burgers $(1950,1974)$. Despite the difference in their spectra, in matters of the spectral energy transfer and triadic interactions Burgers turbulence is similar to Navier-Stokes turbulence.
\end{abstract}

\footnotetext{
"This research was supported by the National Aeronautics and Space Administration under NASA Contract No. NAS1-19480 while the authors were in residence at the Institute for Computer Applications in Science and Engineering (ICASE), NASA Langley Research Center, Hampton, VA 23681-0001.
} 



\section{Introduction.}

Burgers equation (Burgers 1950, 1974) describes the behavior of weak shocks in compressible turbulence and is also considered a one-dimensional model of the Navier-Stokes equations. For these reasons it has received a great deal of attention in the literature. The objective of this paper is to investigate some of the less explored aspects of the spectrum and spectral energy transfer interactions in numerically generated Burgers turbulence (B-turbulence) which is maintained steady by low-wavenumber forcing. In particular, we would like to establish the similarities and differences between B-turbulence and Navier-Stokes turbulence (NS-turbulence) in matters of the energy spectrum and spectral energy transfer. Due to the one-dimensional nature of the velocity field, spectral interactions in B-turbulence are more easily tractable than in NS-turbulence (Domaradzki and Rogallo, 1990; Yeung and Brasseur, 1991; Okhitani and Kida, 1992; and, Zhou, 1993).

The forced Burgers equation under consideration here is given by

$$
\frac{\partial u}{\partial t}+u \frac{\partial u}{\partial x}=\nu \frac{\partial^{2} u}{\partial x^{2}}+f(x, t)
$$

where $\nu$ is kinematic viscosity and $f(x)$ is the forcing term. The above equation is solved pseudospectrally ( $N$ nodes) with periodic boundary conditions from a random initial velocity field. The initial velocity field is specified in the spectral space $(\hat{u}(k, 0))$ as follows:

$$
\hat{u}(k, 0)= \begin{cases}C k^{-n} & \text { for } k_{i} \leq k \leq k_{f} \\ 0 & \text { for other } k,\end{cases}
$$

where $k$ is the wavenumber and $n, C, k_{i}$ and $k_{f}$ are specified values. The phase of each wavenumber is assigned a random value in the range $(0,2 \pi)$. Forcing is performed in the spectral space as follows. At the end of each time step, the amplitudes of wavenumbers in the range $\left(k_{i}, k_{f}\right)$ are reset to their value at the initial time: that is, $\hat{u}(k, t)=C k^{-n}$. The phases associated with these wavenumbers are as determined by the Burgers equation. As for the other wavenumbers, both the amplitude and phase evolve by the Burgers equation. This manner of forcing leads to steady (time-invariant) spectrum and velocity field after an initial transient (period of time). All the results presented below (except those demonstrating the inverse cascade) are calculated after the velocity field reaches a steady state. The values of the parameters for the various cases studied are given in the Table. Simulations are performed for three different values of $n(=0,1 / 2,1)$. The turbulence is forced in the above fashion in the wavenumber range $(1,20)$. For all the cases given in the table, $n=1$. 


\section{Background.}

The classical picture of the NS-turbulence is the following. The turbulent kinetic energy is produced due the interaction between the fluctuating and the mean velocity field at scales of motion classified as the energy containing scales. This energy is then cascaded down (due to triadic interactions) to smaller scales of motion called the inertial scales. There is no production or viscous dissipation of kinetic energy in these scales. The amount of energy cascaded down the inertial scales is given the name spectral energy transfer rate. The kinetic energy is then cascaded down (again by triadic interactions) from the inertial scales to even smaller scales of motion called the dissipation scales. In these scales, viscous action dissipates the kinetic energy. According to the Kolmogorov (1941) hypothesis, the inertial range can be completely characterized by the spectral energy transfer rate and the dissipative scales by the energy dissipation rate and viscosity. In steady state turbulence, the spectral energy transfer rate is equal to the dissipation rate $(\epsilon)$ of the turbulent kinetic energy. This leads to the following spectral behavior in the inertial range

$$
E(k) \sim \epsilon^{\frac{2}{3}} k^{-\frac{5}{3}}
$$

The functional form of the spectrum in the dissipation range has been established only recently using DNS (eg., Kida and Murakami 1987, Sanada 1992, Zhou 1993, Chen et al 1993):

$$
E(k) \sim \exp (-\beta \eta k)
$$

where, $\eta=\left[\frac{\nu^{3}}{\epsilon}\right]^{\frac{1}{4}}$ and $\beta$ is a constant between 5 and 10 .

The spectrum of the Burgers velocity field also exhibits three distinct regimes which for historical reasons will again be called the energy containing range, the inertial range and the dissipation range (Kida, 1981). If the classical picture of NS turbulence is accurate for B-turbulence also, then the Kolmogorov hypothesis would lead to a spectrum similar to that of NS turbulence. However,

- it is known from experience that the inertial range spectrum certainly does not scale as $k^{-\frac{5}{3}}$. Several authors (Burger 1950, Saffman 1968) have suggested a different picture of B-turbulence dynamics that yields the correct (observed) inertial range spectral behavior. Several other aspects of the suggested B-turbulence picture are yet unverified. Also, the nature of the spectral triadic interactions, which may shed more light on the spectral behavior, is yet unexplored. We first 
briefly present some results from the analysis of Burgers $(1950,1974)$ and Saffman $(1968)$ and then proceed to interrogate the numerically generated Burgers velocity field. Comparisons with the NS-turbulence behavior will be made wherever possible.

Qualitative description of Burgers turbulence. The Burgers velocity field is composed of a series of discrete viscous shocks. For a periodic train of shocks, it can be shown (Saffman 1968) that the velocity jump $(V)$ across the shock is given by

$$
V=\sqrt{12 K}=\sqrt{6} u_{r m s} .
$$

where $K$ and $u_{r m s}$ are the kinetic energy and the root mean square of the velocity field. The characteristic shock thickness is of the order

$$
d \sim \frac{4 \nu}{V}
$$

The shock structure can be completely parametrized in terms $V$ and the shock separation length $L$, which is approximately the velocity auto-correlation length scale. All of the dissipation occurs inside these viscous shocks. The smallest length scales (dissipation scales) are of the order of the shock thickness, $d$. The small scale statistics of the velocity field are governed by the inner shock structure. Analysis of the shock structure yields the following: the dissipation rate is given by

$$
\epsilon=\frac{V^{3}}{24 L}
$$

and, the dissipation-range spectrum has the form

$$
E(k)=\frac{2 \nu^{2} \pi}{L} \exp \left(-\frac{\pi \nu}{V} k\right)
$$

It is clear then that, as in NS turbulence, the kinetic energy dissipation rate $(\epsilon)$ is independent of viscosity. However, unlike NS turbulence where the small scales are completely characterized by $\epsilon$ and $\nu$, the small structure of B-turbulence is determined by $V$ and $L$ which are large scale parameters.

In the classical Burgers equation analysis, the inertial-range scales $\left(k_{f} \ll k \ll \frac{2 \pi}{d}\right.$, where $k_{f}$ is the largest forced wavenumber) are treated as inviscid. The inner shock structure is deemed unimportant for determining the statistics of these scales. For the purpose of analyzing these 
inertial scales, the finite-thickness viscous shocks are treated as infinitesimally thin discontinuities in the velocity field. These simplifications, then, lead to the well known spectrum of a collection of discontinuities for the inertial range

$$
E(k) \sim k^{-2}
$$

\section{Results.}

Inertial-range spectrum. Although the inertial-range behavior of the Burgers spectrum is well established in previous studies, we present the spectra from our computations here to demonstrate the validity of our forcing technique and the numerics. For all the values of the initial spectrum exponent $(n)$ considered, the energy spectrum of the numerically generated velocity field does yield a $k^{-2}$ behavior in the inertial range. The spectrum exhibits the smoothest characteristics for $n=1$, which corresponds to $E(k) \sim k^{-2}$ in the forced range. In fact, for this value of $n$, the boundary between the forced range and the inertial range is indistinguishable. The compensated spectra computed from various simulations (with $n=1$, but different Reynolds numbers characterized by different values of $C$ and $\nu$ ) are shown in figure 1. The so-called compensated spectrum is obtained by pre-multiplying the the energy spectrum by $k^{2}$. The compensated spectra for the various simulations are flat for over a decade past the forcing scales, demonstrating the $k^{-2}$ behavior in the inertial range.

Dissipation-range spectrum. The spectral behavior in the dissipation range (of B-turbulence) is yet unexamined in the numerical studies in literature. We now compare the theoretically predicted spectrum given in equation (8) with simulation data. In figure $2 \mathrm{~b}$, the results of the simulations are shown in a log-linear plot with the energy spectrum and wavenumber normalized with the scaling shown in equation (8). The various spectra collapse very well along a (-1)-slope line, indicating good agreement between theory and simulations. For the sake of comparison, the degree of agreement between the simulation data and Kolmogorov scaling (equation 4) is also investigated. In figure $2 \mathrm{a}$, a log-linear plot of the energy spectra is provided with the wavenumber normalized by $\eta$, the Kolmogorov length scale. As can be seen from the figure, the collapse of the various spectra is poor. This is to be expected since, unlike in the case of NS-turbulence, the dissipation rate and viscosity do not exclusively determine the statistical behavior of small scales in B-turbulence. 
Spectral energy transfer budget. In spectral space, the Burgers equation for the unforced wavenumbers can be written as

$$
\frac{\partial \hat{u}(k) \hat{u}^{*}(k)}{\partial t} \equiv \frac{\partial \hat{E}(k)}{\partial t}=T(k)-D(k)
$$

The term $D(k) \equiv 2 \nu k^{2} \hat{E}(k)$ represents the rate at which energy is dissipated by wavenumber $k$. The net rate at which energy is input into wavenumber $k$ is given by $T(k) \equiv \sum_{p, q} T(k, p, q)$ with

$$
\begin{aligned}
T(k, p, q) & =-i k \times \operatorname{Im}\left[\hat{u}^{*}(k) \hat{u}(p) \hat{u}(q)\right] \delta(k-p-q) \\
& =-i k \times \operatorname{Im}\left[\hat{u}^{*}(k) \hat{u}(p) \hat{u}(k-p)\right] \equiv T(k, p)
\end{aligned}
$$

In the above equations, superscript ${ }^{*}$ and $I m$ represent the complex conjugate and imaginary parts of a complex number, and $\delta()$ stands for the Dirac delta function. The term $T(k, p, q)$ is the energy transfer into wavenumber $k$ due to the triadic interaction between the wavenumbers $k, p$ and $q$. A positive value of $T(k, p, q)$ indicates that the triadic interaction adds energy to the wavenumber $k$ and a negative value denotes removal of energy.

The spectral energy transfer equation in incompressible Navier-Stokes turbulence looks exactly similar to that of B-turbulence (equation 10) with two exceptions: (i) the wavenumbers $k, p$ and $q$ are vectors rather than scalars; and (ii) the triadic energy transfer function is given by

$$
\dot{T}(\mathbf{k}, \mathbf{p}, \mathbf{q})=-\frac{1}{2} i \operatorname{Im}\left[P_{a b c}(\mathbf{k}) \hat{\mathbf{u}}_{\mathbf{a}}^{*}(\mathbf{k}) \hat{\mathbf{u}}_{\mathbf{b}}(\mathbf{p}) \hat{\mathbf{u}}_{\mathbf{c}}(\mathbf{q})\right] \delta(\mathbf{k}-\mathbf{p}-\mathbf{q})
$$

where

$$
P_{a b c}(\mathbf{k})=k_{b}\left(\delta_{a c}-\frac{k_{a} k_{c}}{k^{2}}\right)+k_{c}\left(\delta_{a b}-\frac{k_{a} k_{b}}{k^{2}}\right)
$$

In stationary isotropic NS-turbulence and in steady B-turbulence, the spectral transfer term $T(k)$ and the dissipation term $D(k)$ balance one another other at each wavenumber. In NavierStokes turbulence, the magnitude of $D(k)$ (and hence that of $T(k)$ ) is small in the inertial range increasing to a peak in the dissipation range before ultimately going to zero at higher wavenumbers. The spectral energy budget of B-turbulence (at the non-forced wavenumbers) is shown in figure 3a. The behavior of $D(k)$ in this case is completely different from that in NS-turbulence. The magnitude of $D(k)$ (and hence of $T(k)$ ) is almost constant at a high value in the inertial range and then decays monotonically to zero in the dissipation range. That is, unlike NS-turbulence. turbulent kinetic energy is dissipated by inertial range scales also, at a rate larger than that of the 
so-called 'dissipation range' scales. This is clearly the reason why, unlike in NS-turbulence, the dissipation rate of turbulent kinetic energy $\epsilon$ (or the spectral transfer rate) cannot solely determine the state of inertial range. This behavior gives rise to an important question: how is it that the inviscid analysis predicts the correct behavior of the spectrum in the inertial range, and yet, viscous dissipation rate of the inertial range is larger than that in the dissipation range? To address this question we examine the behavior of two quantities $T^{+}(k)$ and $T^{-}(k)$ defined as

$$
T^{+}(k)=\sum_{p, q} \max \{T(k, p, q), 0\} ; T^{-}(k)=\sum_{p, q} \min \{T(k, p, q), 0\}
$$

leading to $T(k)=T^{+}(k)+T^{-}(k)$. ¿From the definitions it is clear that $T^{+}(k)$ is the energy influx, $T^{-}(k)$ is the energy efflux, and $T(k)$ is the net energy flux into $k$ from all of the triadic interactions. Also from the definition, it is seen that the spectral energy transfer rate $\Pi(k)=\left|T^{-}(k)\right|$. The quantities $T^{+}, T^{-}$and $T$ are given in figure $3 \mathrm{~b}$ as a function of $k$. The magnitudes of $T^{+}(k)$ and $T^{-}(k)$ suffer a substantial monotonic decay in the inertial range. Despite the decay, the magnitudes of $T^{+}$and $T^{-}$in the inertial range of B-turbulence are sitll much larger than that of $T(k)$ and, hence, that of $D(k)$. Therefore, although the dissipation $(D(k))$ in the inertial range is larger than that in the far range, the $D(k)$ in the inertial range is very small compared to the magnitudes of energy influx and efflux. As a result, the treatment of the inertial scales as inviscid is a reasonable approximation. In the far range, the magnitudes of $D\left(K^{-}\right), T^{+}(k)$ and $T^{-}(k)$ are nearly equal, and hence, viscous effects are as important as triadic energy transfer or the non-linear effect.

The demonstrated behavior of $T^{+}$and $T^{-}$in the inertial range of B-turbulence is very different from that in NS-turbulence where one would expect to see $T^{+}(k)=T^{-}(k) \approx$ Constant, resulting in the spectral transfer rate being equal to the total dissipation rate.

Are the above differences between B-turbulence and NS-turbulence reflected in the nature of triadic interactions? In order to answer this question, we examine the spectral energy transfer and locality of the most intense and most net-energy-transfer triadic interactions.

Spectral energy transfer. The nature of spectral energy transfer is best understood by examining the function $T(k, p) \equiv \sum_{q} T(k, p, q)$. The net energy transfer from a wavenumber $p$ to a wavenumber $k$ is given by $T(k, p)$. A negative value of $T(k, p)$ indicates net energy transfer from $k$ to $p$, whereas a positive value indicates a net transfer of energy from $p$ to $k$. In figure $4 a, T(k, p)$ 
is shown as function of $k$ for a value of $p=50$ which is in the inertial range. (In B-turbulence, due to one-dimensionality of the problem, we have $T(k, p)=T(k, p, q)$, where $q=k-p$.) The value of $T(k, p)$ is negative for all $k<p$. At small $k$, values $T(k, p)$ is small in magnitude. It decreases monotonically to a minimum at $k=p-1$. For $p=k, T(k, p)$ is zero. For all $k>p, T(k, p)$ is positive. It has the highest value for $k=p+1$, and then decreases monotonically to zero for higher $k$ values. Similar behavior is observed for all non-forced $p$ values examined (figures not shown). From the figure shown, the following picture of the energy transfer emerges. A given scale of motion draws energy from larger scales. The highest energy is drawn from the scale that is the next larger size. Energy is then lost to smaller scales. The most energy is lost to the scale that is the next smaller size. This type of energy flow from large to small scales in a continuous manner is given the name 'energy cascade'. The behavior of $T(k, p)$ in NS-turbulence (Domaradzki and Rogallo 1990. Ohkitani and Kida 1992, Yeung and Brasseur 1991, and Zhou 1993) is quite similar to that shown in Figure 4a. Therefore, the conclusion from figure $4 \mathrm{a}$ is, the energy transfer in B-turbulence is local in wavenumber space, implying energy cascade, as is the case in NS-turbulence.

Locality of the energetic triadic interactions. The energy transfer between wavenumbers $k$ and $p$ occurs due to the interaction between three wavenumbers: $k, p$ and $q$. Often, it is of interest to know if a particular triadic interaction is local or non-local in nature. Local interactions are interactions between three neighbouring wavenumbers that are of similar magnitude. Non-local interactions invovle wavenumbers of vastly disparate magnitudes. One of the most commonly used metrics of the locality of the interaction between a given set of wavenumbers is the scale disparity parameter $S(k, p, q)$ :

$$
S(k, p, q)=\frac{\max \{k, p, q\}}{\min \{k, p, q\}} . \equiv \frac{\max \{k, p, k-p\}}{\min \{k, p, k-p\}} . \equiv S(k, p) .
$$

Small values of $S(<20$, approximately) indicate local triadic interactions and larger values indicate distant or non-local interactions (Zhou 1993).

Presented in figure $4 \mathrm{~b}$ are the $S$ values of the triadic interactions that produce the energy transfer shown in figure $4 \mathrm{a}$. The values of the $j$ wavenumber participating in the triadic interactions are also shown in figure $4 \mathrm{~b}$. ¿From figure $4 \mathrm{a}$, it is clear that the energy transfer between wavenumber $p=50$ and its neighboring $k$ values are the most intense. This intense energy transfer is brought about due 
to the triadic interaction between $k \approx 50, p=50$ and $q=k-p \approx 1$. The scale disparity parameter corresponding to these intense interactions is $S \approx 50$. For all values of $p$ considered (figures not shown), the scale disparity parmeter for the most intense triadic interactions is $S \approx p$. Clearly, the most energetic triadic interactions always will involve the small wavenumbers or the large scales. Hence, the most energetic interactions in B-turbulence are non-local, as in NS turbulence (Domaradzki and Rogallo 1990, Ohkitani and Kida 1992, Yeung and Brasseur 1991, and Zhou $1993)$.

Locality of the highest net-energy-transfer interactions. In NS-turbulence, while some distant interactions transfer large amounts of energy into a wavenumber, other distant interactions remove equally large amounts of energy from that wavenumber. As a result, when the contributions of all the distant interactions are added up, the net energy transfer into any wavenumber is very small. Dynamically significant amounts of net energy transfer into the inertial scales come from local interactions (Zhou 1993). In NS-turbulence, this realization is crucial for reconciling the observed non-locality of the most energetic interactions with the Kolmogorov 1941 hypothesis, which hinges on the assumption that only local triadic interactions are important in the inertial range. We now examine if the net energy transfer in B-turbulence is from local or distant interactions. The net energy transfer into a wavenumber $k$ from interactions of a particular locality $S$ is given by,

$$
T(k, S)=\sum_{p, q} T(k, p, q) \delta[S-S(k, p, q)]
$$

Rather than considering contributions from individual $S$ values, net energy transfer to wavenumber $k$ from logarithmic bands of $S$ values are considered:

$$
T\left(k, S_{n}\right)=\sum_{S=2^{n}}^{S=2^{n}+1} T(k, S) .
$$

In figure 5, the net energy transfer from each $S$-band (as a percentage of the total net energy transfer) into a wavenumber $k$ is plotted as a function of the central $S$ value of the band. Four different $k$ values $(75,100,200.500)$ located in inertial and dissipation ranges are considered. In each case, more than $50 \%$ of the net energy transfer is from $S$ values under four. More than $80 \%$ of the net energy transfer is from local triadic interactions $(S<20)$. The conclusion from figure 5 is, then, as in NS-turbulence, the local interactions transfer most net energy. 
Inverse Cascade. It has long been known that three-dimensional NS-turbulence does not permit the inverse cascade of energy from small scales to large scales, whereas, two-dimensional turbulence does (Kraichnan 1967, Pouquet et al 1975). The analysis of Kraichnan (1968) indicates that the Burgers equation may permit inverse cascade. which, we now attempt to verify using numerically generated data. We perform a simulation with no forcing and with the initial field specified according to equation (2) with $n=1, k_{i}=21$ and $k_{f}=50$. All wavenumbers outside the range (21. 50) are initially unenergetic. The evolution of the spectrum of this decaying velocity field is shown in figure 6. As the initial field evolves according to the Burgers equation, all wavenumber modes, those smaller and larger than the initially energetic ones, acquire kinetic energy very rapidly. Hence, the energy transfer from the initially energetic scales to larger scales is evident. After this rapid transient stage, the spectrum decays with no more evidence of inverse energy transfer. With time, the larger wavenumbers dissipate their energy more rapidly than the smaller ones. In fact, during the later stages of decay, only the $k<20$ nodes are energetic.

The rapid acquisition of energy by large scales $(k<20)$ is a unequivocal demonstration of inverse energy transfer. However, it is not at all clear if this transfer happens in the form of a cascade, a question which can be answered only by analyzing the triadic interactions. The analysis of the triadic interactions during inverse transfer is rendered difficult by the fleeting nature of that process and is deferred to a future study. After the initial transient period, the direction of energy transfer is from large to small scales and the nature of the triadic interactions at this stage is similar to that discussed in detail in figures $3-5$.

\section{Summary and Conclusion.}

Using numerically generated B-turbulence data it has been established that: (i) in the inertial range, the spectrum scales as $k^{-2}$ : (ii) in the dissipation range, the large scale parameters $L$ and $V$ collapse the spectra very well; (iii) although the inertial range scales dissipate turbulent kinetic energy more rapidly than the far range, inviscid analysis still yields an accurate scaling of the inertial range spectra: (iv) energy transfer is local in spectral space; (v) the triadic interactions causing the most energetic transfers are distant: and (vi) the triadic interactions causing most net energy tranfer are local. On the whole, the spectral energy transfer and triadic interactions in 
Burgers turbulence are quite similar to those in NS turbulence, despite major differences in the mechanics (spectral scaling in the inertial and far ranges) of the two equations.

\section{References}

[1] Burgers, J. M., (1950) Correlation problems in a one-dimensional model of turbulence I - IV. Proc. Acad. Sci. Amsterdam, 53, 247-260, 393-406, 718-742.

[2] Burgers, J. M., (1974) The non-linear diffusion equation. Reidel, Dordrecht.

[3] Chen, S., Doolen, G., Herring, J. R., Kraichnan, R. H., Orszag, S. A., and She, Z. E., (1993) Far-dissipation range of turbulence. Phys. Rev. Lett, 70, 3051

[4] Domaradzki, J. A., and Rogallo, R. S., (1990) Local energy transfer and non-local interactions in homogeneous isotropic turbulence. Phys. Fluids A, 2, 413.

[5] Kida, S., (1981) Similar solutions in modified cumulant expansion. Phys. Fluids, 24 (4), 604 614.

[6] Kida, S., Murakami, Y., (1987) Similar solutions in modified cumulant expansion. Phys. Fluids, 30, 2030 .

[7] Kraichnan, R. H., (1967) Inertial ranges in two-dimensional turbulence. Phys. Fluids, 10, 1417.

[8] Kraichnan, R. H., (1968) Lagrangian history statistical theory for Burgers turbulence. Phys. Fluids, 11, 265.

[9] Ohkitani, K., and Kida, S., (1992) Triad interactions in a forced turbulence. Phys. Fluids A, 4,794 .

[10] Pouquet, A., Lesieur, M., Andre, J. C., and Basdevant, C., (1975) Evolution of high Reynolds number two-dimensional turbulence. J. Fluid Mech., 72, 305.

[11] Saffman, P. G., (1968) Lecture on homogeneous turbulence. In 'Topics in non-linear Physics'. Ed. N. J. Zabusky, Springer.

[12] Sanada, T., (1992) Comments on the dissipation-range spectrum in turbulent flows. Phys. Fluids A, 4, 1086. 
[13] Yeung, P. K., and Brasseur, J. G., (1991) The response of isotropic turbulence to isotropic and anisotropic forcing at large scales. Phys. Fluids A, 3, 884.

[14] Zhou, Y., (1993) Interacting scales and energy transfer in isotropic turbulence. Phys. Fluids A, 5, 2511 .

Table

\begin{tabular}{|l|c|c|c|c|c|c|c|c|c|c|c|}
\hline \hline Cases & $N$ & $k_{i}$ & $k_{f}$ & $C$ & $v$ & $\left\langle u^{2}\right\rangle$ & $\left(\left(\frac{\partial u}{\partial x}\right)^{2}\right)$ & $V$ & $L$ & $u_{\eta}$ & $\eta$ \\
\hline 1 & $2^{14}$ & 1 & 20 & 2 & .01 & 1.09 & 62.19 & 2.55 & 1.114 & 0.2808 & 0.0356 \\
2 & $2^{14}$ & 1 & 20 & 2 & .005 & 1.09 & 121.22 & 2.56 & 1.149 & 0.2346 & 0.0213 \\
3 & $2^{14}$ & 1 & 20 & 3 & .01 & 1.25 & 206.00 & 3.87 & 1.174 & 0.3788 & 0.0264 \\
4 & $2^{15}$ & 1 & 20 & 5 & .01 & 3.40 & 947.34 & 6.40 & 1.151 & 0.5547 & 0.0180 \\
\hline
\end{tabular}




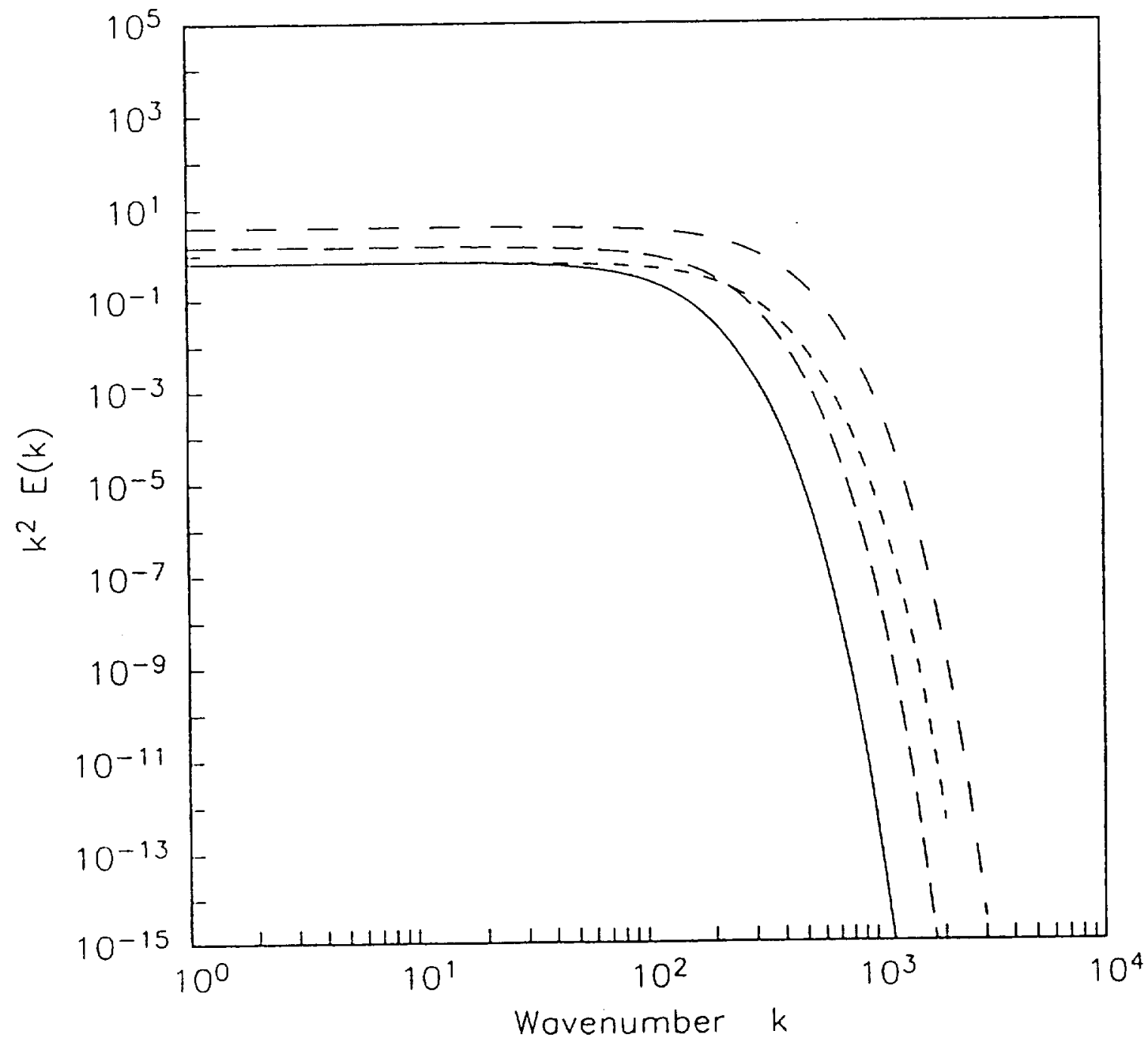

Figure 1: Compensated spectra $\left(k^{2} E(k)\right)$ vs. $\mathrm{k}$ for various cases: solid line - Case 1; (- -) Case 2; (- -) Case 3 ; and, (- -$)$ Case 4. 
Figure 2a

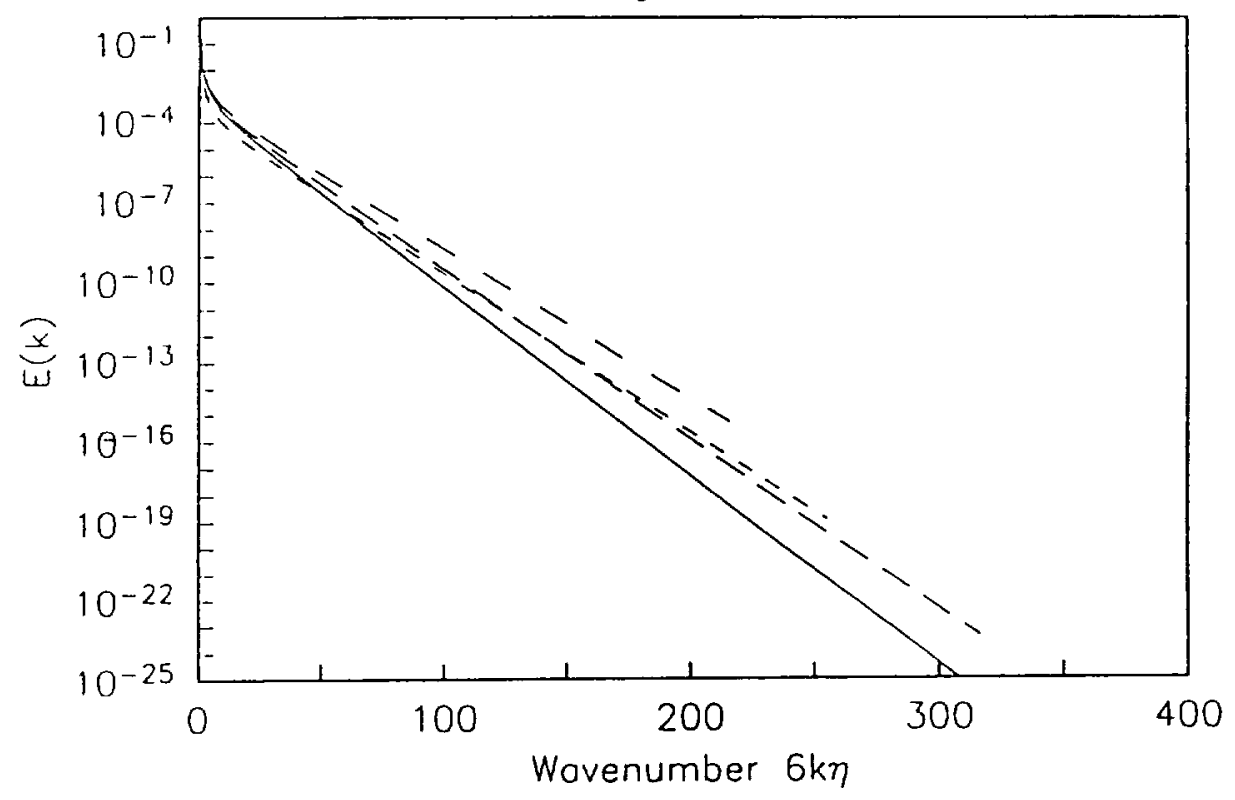

Figure $2 b$

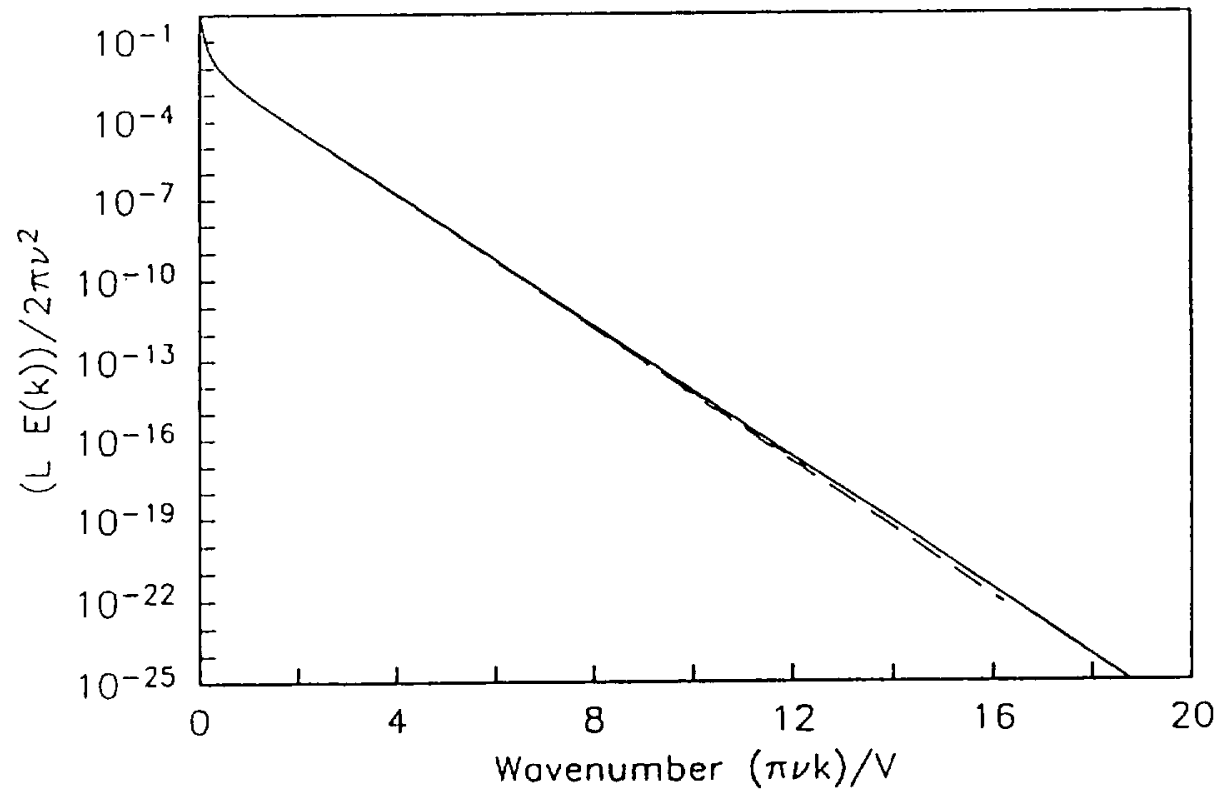

Figure 2: Dissipation range spectra for various cases. Figure $2 \mathrm{a}$ is with Kolmogorov scalings and Figure $2 \mathrm{~b}$ with Saffman scaling. Legend same as figure 1. 
Figure 3a

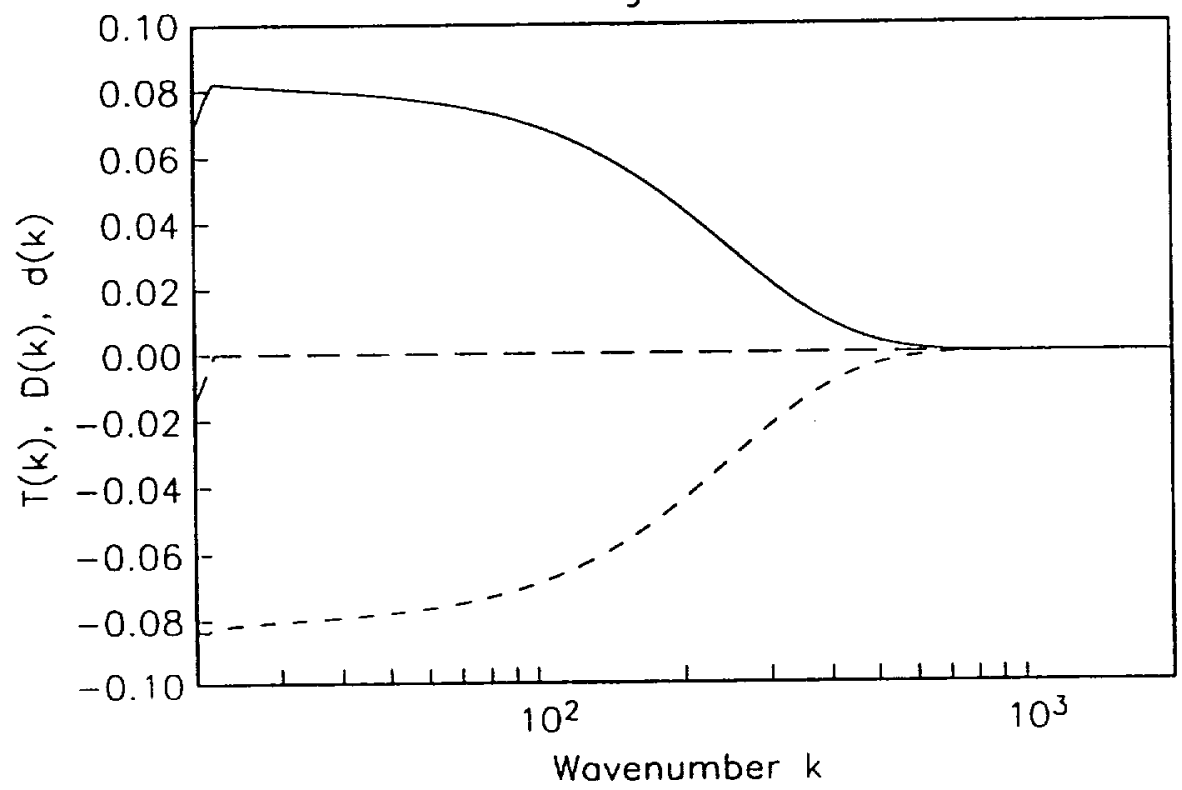

Figure 3b

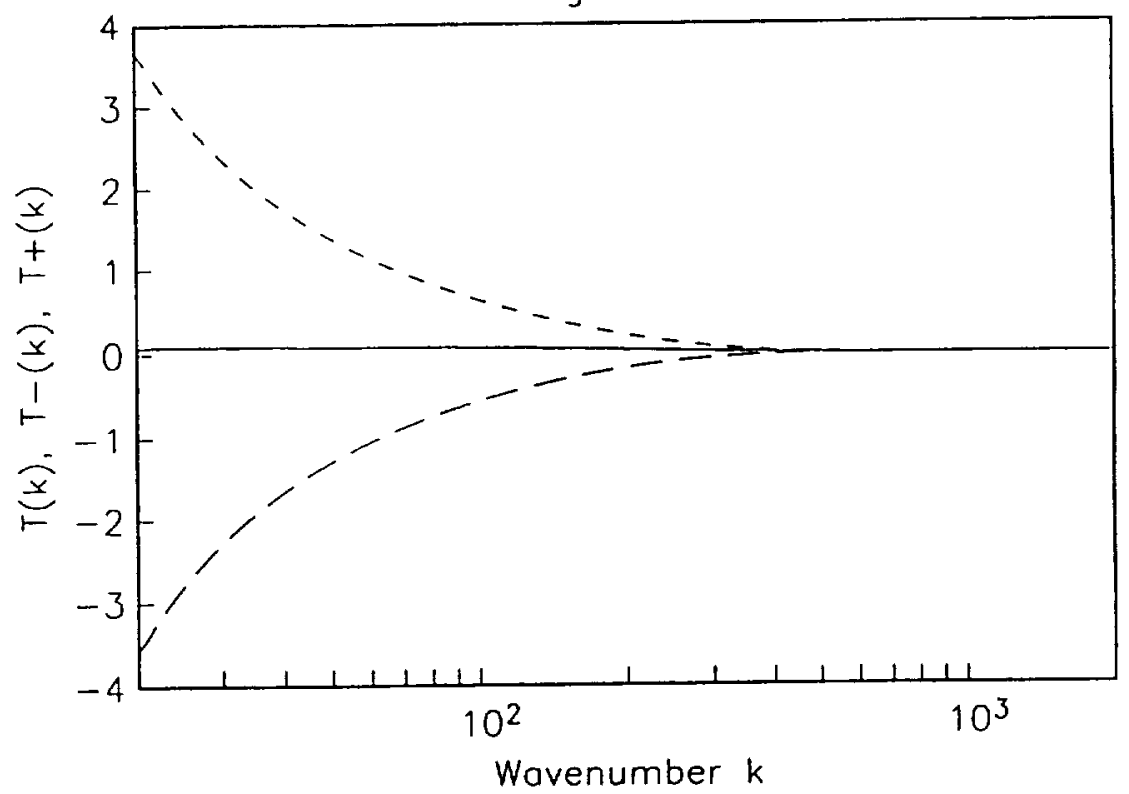

Figure 3: Spectral energy transfer budget for Case 4. Figure 3a: solid line $-T(k) ;(--)-D(k)$; and, $(--)-d(k)=T(k)-D(k)$. Figure 3 b: solid line $-T(k) ;(--)-T^{+}(k)$; and,$(--)-T^{-}(k)$. 
Fig. 40

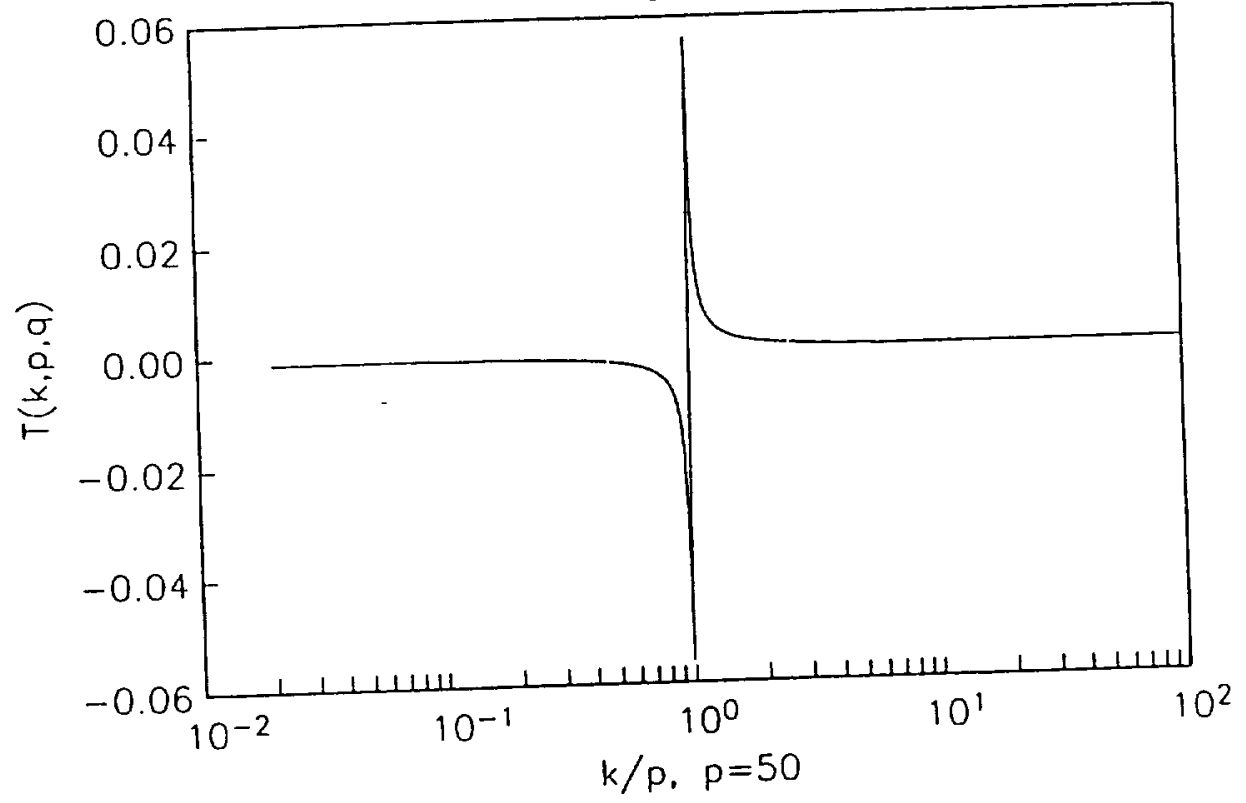

Fig. $a b$

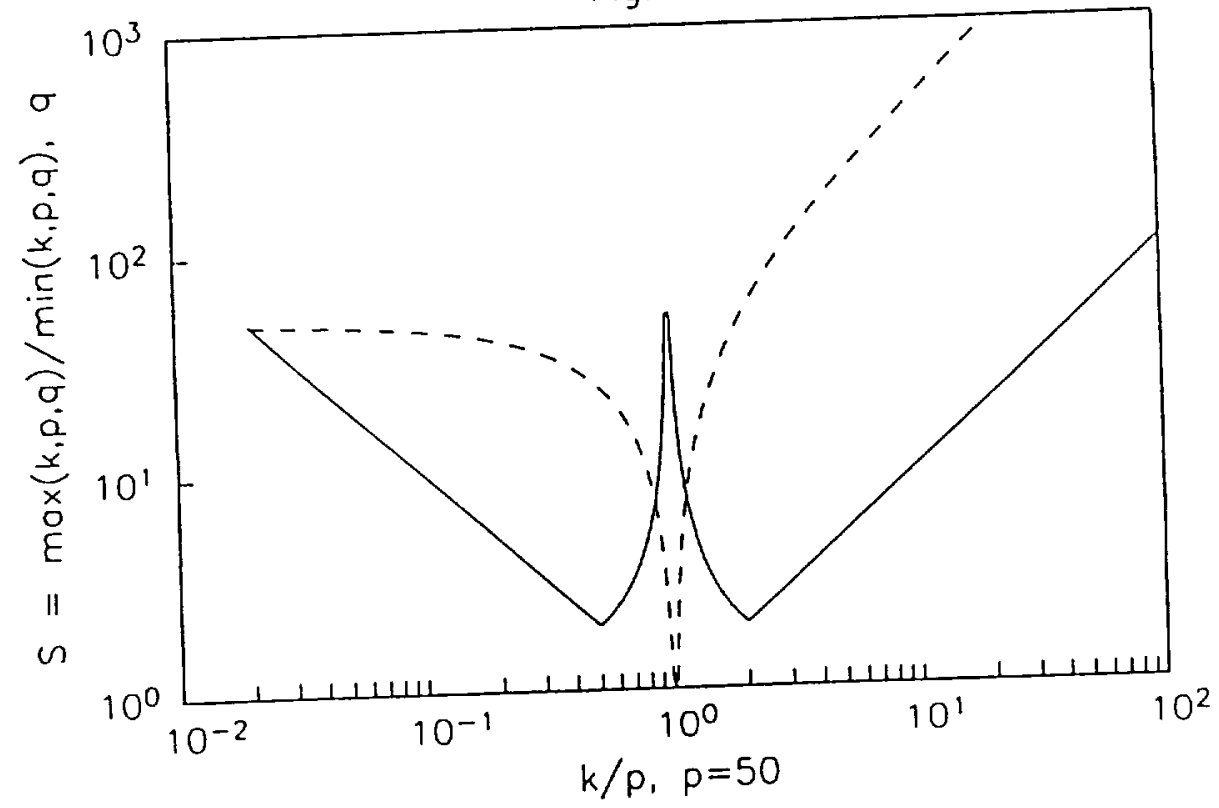

Figure 4: Triadic interactions for Case 4. Figure 4a: $T(k, p, q)$. Figure $4 b: S(k, p, q)$ - solid line, $q$ dashed line. 
Figure 5

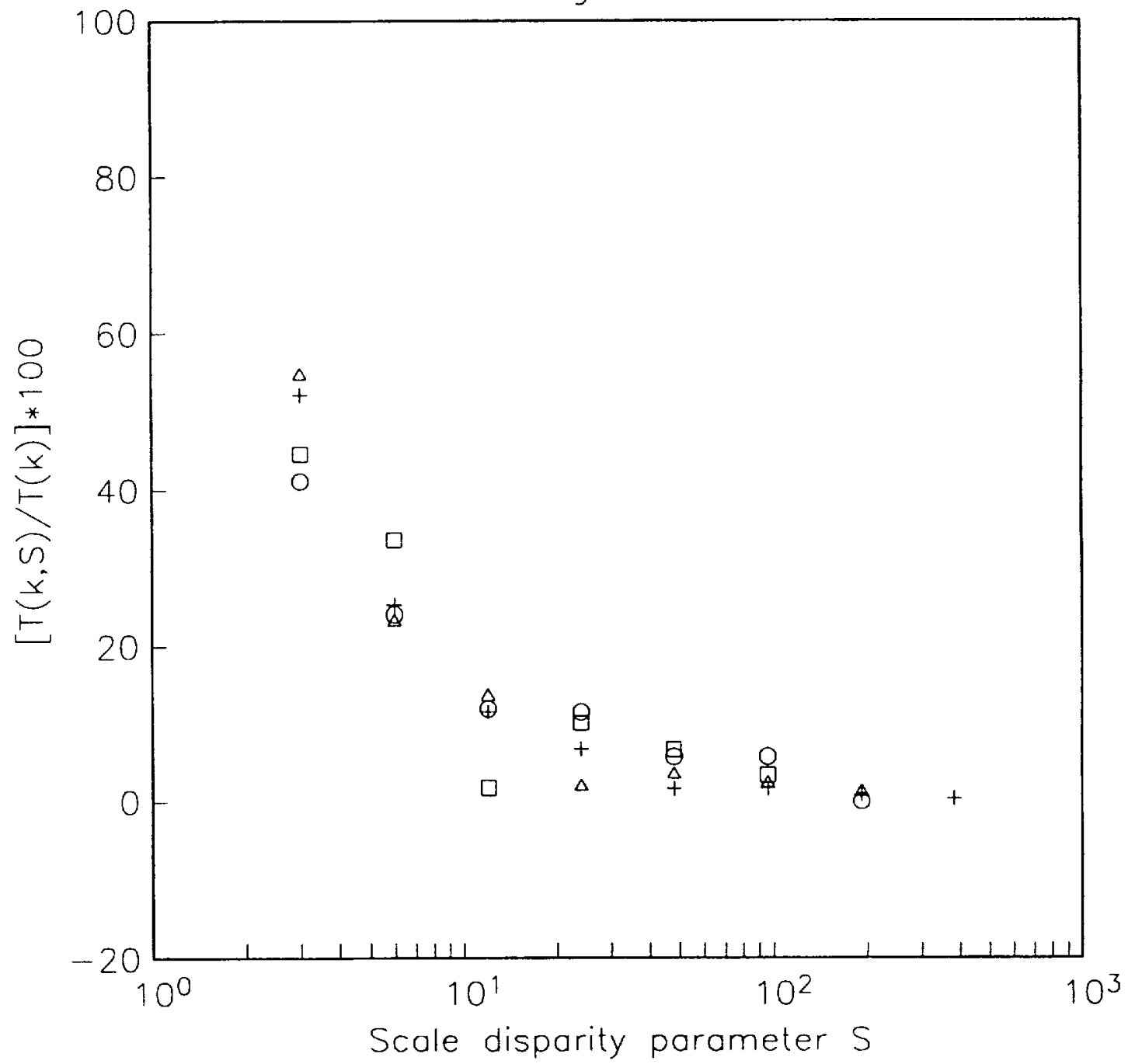

Figure 5: Locality of most net energy transfer interactions. $\left[T\left(k, S_{n}\right) / T(k)\right] * 100$ vs. $S . k=75$ $(\bigcirc), 100(\square), 200(\triangle)$ and $500(+)$. 


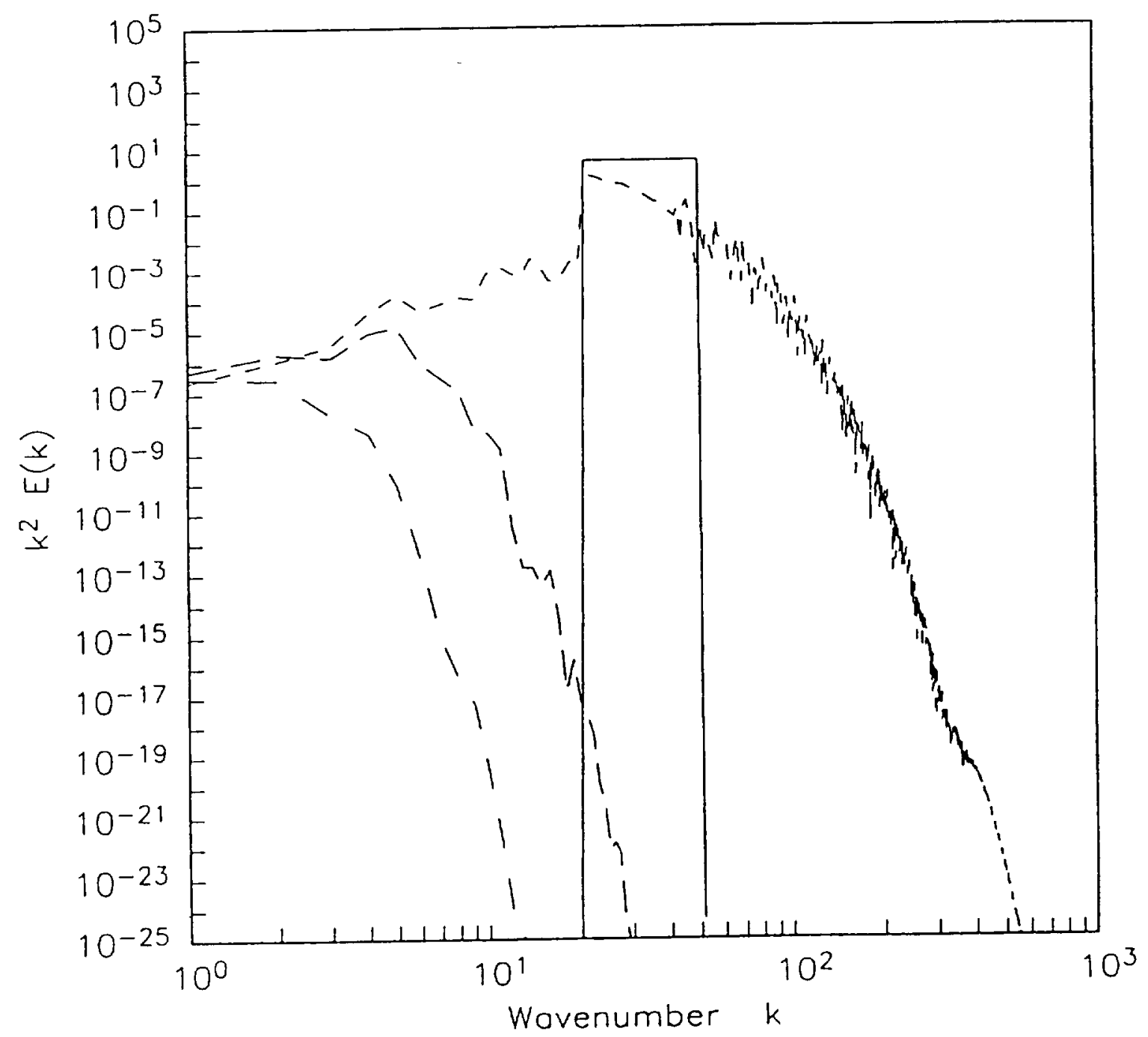

Figure 6: Inverse cascade demonstration. Spectrum of decaying Burgers field at various times. For this case $k_{i}=20, k_{f}=50, C=1$ and $\nu=0.02$ and no forcing. The various times are: $\mathrm{t}=0$ (solid line); $\mathrm{t}=.06(--) ; \mathrm{t}=3(--)$; and, $\mathrm{t}=15(--)$. 
Public reporting burden for this collection of information is estimated to average 1 hour per response, including the time for reviewing instructions, searching existing data sources,

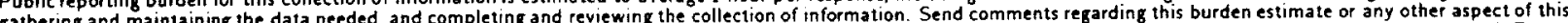
gathering and maintaining the data needed, and compluing a burden. to Washington Headquarters Services. Directorate for Information Operations and Reports. 1215 Jefferson Davis Highway, Suite 1204 Arlington, VA 22202-4302, and to the Office of Management and Budget. Paperwork Reduction Project (0704-0188). Washington, DC 20503.

\begin{tabular}{|l|l|l}
\hline 1. AGENCY USE ONIY(Leave blank) & $\begin{array}{l}\text { 2. REPORT DATE } \\
\text { March } 1995\end{array}$ & $\begin{array}{l}\text { 3. REPORT TYPE AND DATES COVERED } \\
\text { Contractor Report }\end{array}$ \\
\hline
\end{tabular}

4. TITLE AND SUBTITLE SPECTRUM AND ENERGY TRANSFER IN STEADY BURGERS TURBULENCE

C NAS1-19480

WU 505-90-52-01

6. AUTHOR(S)

Sharath S. Girimaji

Ye Zhou

7. PERFORMING ORGANIZATION NAME(S) AND ADDRESS(ES)

Institute for Computer Applications in Science

and Engineering

Mail Stop 132C, NASA Langley Research Center

Hampton, VA 23681-0001

9. SPONSORING/MONITORING AGENCY NAME(S) AND ADDRESS(ES)

National Aeronautics and Space Administration

Langley Research Center

Hampton, VA 23681-0001

8. PERFORMING ORGANIZATION

REPORT NUMBER

ICASE Report No. 95-13

11. SUPPLEMENTARY NOTES

Langley Technical Monitor: Dennis M. Bushnell

Final Report

Submitted to Physics Letters A

12a. DISTRIBUTION/AVAILABILITY STATEMENT

12b. DISTRIBUTION CODE

Unclassified-Unlimited

Subject Category 34

13. ABSTRACT (Maximum 200 mords)

The spectrum, energy transfer and spectral interactions in steady Burgers turbulence are studied using numerically generated data. The velocity field is initially random and the turbulence is maintained steady by forcing the amplitude of a band of low wavenumbers to be invariant in time, while permitting the phase to change as dictated by the equation. The spectrum, as expected, is very different from that of Navier-Stokes turbulence. It is demonstrated, for the first time, that the far range of the spectrum scales as predicted by Burgers $(1950,1974)$. Despite the difference in their spectra, in matters of the spectral energy transfer and triadic interactions Burgers turbulence is similar to Navier-Stokes turbulence.

\begin{tabular}{|c|c|c|c|}
\hline \multicolumn{3}{|c|}{$\begin{array}{l}\text { 14. SUBJECT TERMS } \\
\text { Burgers Turbulence; Triadic Interactions; Spectral Energy Transfer; Burger Spectrum }\end{array}$} & \multirow{2}{*}{$\begin{array}{l}\text { 15. NUMBER OF PAGES } \\
19 \\
\text { 16. PRICE CODE } \\
\text { AO3 }\end{array}$} \\
\hline & & & \\
\hline $\begin{array}{l}\text { 17. SECURITY CLASSIFICATION } \\
\text { OF REPORT } \\
\text { Unclassified }\end{array}$ & $\begin{array}{l}\text { 18. SECURITY CLASSIFICATION } \\
\text { OF THIS PAGE } \\
\text { Unclassified }\end{array}$ & $\begin{array}{l}\text { 19. SECURITY CLASSIFICATION } \\
\text { OF ABSTRACT }\end{array}$ & $\begin{array}{l}\text { 20. LIMITATION } \\
\text { OF ABSTRACT }\end{array}$ \\
\hline
\end{tabular}

